Cahiers $d u$ MONDE RUSSE

\section{Cahiers du monde russe}

Russie - Empire russe - Union soviétique et États indépendants

$43 / 2-3 \mid 2002$

Contacts intellectuels, réseaux, relations internationales

\title{
Nicolas Ier et la politique intérieure de la France à l'époque de la Restauration : deux épisodes
}

\author{
Vera MIL'ČINA
}

\section{(2) OpenEdition}

\section{Journals}

Édition électronique

URL : https://journals.openedition.org/monderusse/8500

DOI : 10.4000/monderusse. 8500

ISSN : $1777-5388$

Éditeur

Éditions de l'EHESS

\section{Édition imprimée}

Date de publication : 1 avril 2002

Pagination : 355-374

ISBN : 2-7132-1781-4

ISSN : $1252-6576$

Référence électronique

Vera MIL'ČINA, "Nicolas ler et la politique intérieure de la France à l'époque de la Restauration deux épisodes ", Cahiers du monde russe [En ligne], 43/2-3 | 2002, mis en ligne le 01 janvier 2007, consulté le 03 septembre 2022. URL : http://journals.openedition.org/monderusse/8500 ; DOI : https://doi.org/10.4000/monderusse.8500 


\section{CAIR N}

chercher : repérer : avancer

Cet article est disponible en ligne à l'adresse :

http://www.cairn.info/article.php?ID REVUE=CMR\&ID NUMPUBLIE=CMR 432\&ID ARTICLE=CMR 4320355

Nicolas ler et la politique intérieure de la France à l'époque de la Restauration : deux épisodes

par Vera MIL'CINA

\section{| Editions de l'EHESS | Cahiers du monde russe}

\section{2/2-3 - Vol 43}

ISSN 1252-6576 | ISBN 2713217814 | pages 355 à 374

Pour citer cet article :

- MIL'CINA V., Nicolas ler et la politique intérieure de la France à l'époque de la Restauration : deux épisodes, Cahiers du monde russe 2002/2-3, Vol 43, p. 355-374.

Distribution électronique Cairn pour les Editions de l'EHESS.

(C) Editions de l'EHESS. Tous droits réservés pour tous pays.

La reproduction ou représentation de cet article, notamment par photocopie, n'est autorisée que dans les limites des conditions générales d'utilisation du site ou, le cas échéant, des conditions générales de la licence souscrite par votre établissement. Toute autre reproduction ou représentation, en tout ou partie, sous quelque forme et de quelque manière que ce soit, est interdite sauf accord préalable et écrit de l'éditeur, en dehors des cas prévus par la législation en vigueur en France. Il est précisé que son stockage dans une base de données est également interdit. 


\section{NICOLAS Ier ET LA POLITIQUE INTÉRIEURE DE LA FRANCE À L'ÉPOQUE DE LA RESTAURATION : DEUX ÉPISODES}

Parmi les sources historiques existantes, les relations diplomatiques possèdent une valeur bien rare, à savoir leur synchronie quasi complète avec les événements décrits, permettant ainsi d'éliminer considérablement la déformation ultérieure des choses vues ou entendues. Qui plus est, au XVIII et au XIXe siècle, certains ambassadeurs des pays représentés à la cour russe entretenaient des relations proches, voire intimes, avec les souverains, ce qui confère un caractère d'authenticité unique aux monologues impériaux relatés dans leurs dépêches ${ }^{1}$.

Or, le corpus immense des relations diplomatiques russes et françaises de la Restauration et de la monarchie de Juillet reste en grande partie inédit. (Il en est autrement des rapports du XVIII siècle et du Premier Empire - surtout ceux des envoyés français -, amplement publiés). En ce qui concerne les diplomates russes à l'étranger, leurs correspondances parurent (d'ailleurs, souvent d'une manière fragmentaire) dans le cadre de la série en multiples volumes Vnešnjaja politika Rossii ( $\mathrm{La}$ politique extérieure de la Russie)2 ${ }^{2}$. Cette édition de référence est aujourd'hui arrivée à la première moitié de l'année 1830. Quant au Français Prosper de Barante, qui repré-

1. Le baron de Bourgoing écrivit ainsi à propos de son collègue Auguste Pierre Marie Fenon, comte de La Ferronnays (1777-1842), qui fut l'ambassadeur de France à Saint-Pétersbourg entre 1819 et 1827 (en parlant d'ailleurs des conversations de ce dernier, non avec Nicolas Ier, mais avec son prédécesseur, Alexandre Irr) : «Il est arrivé plusieurs fois que M. de La Ferronnays, après avoir écrit de mémoire tout le discours qu'il venait d'entendre, le soumettait à l'Empereur avant de le transmettre à Paris. Ce prince lisait avec une certaine satisfaction ce qu'il avait dit la veille, et lui savait beaucoup de gré de l'extrême fidélité de sa mémoire, qui lui permettait de rapporter d'aussi longues considérations politiques dans toute leur élégante pureté. Plusieurs fois même, l'Empereur apostilla de sa main la dépêche de l'ambassadeur de France, pour certifier que sa pensée avait été exactement reproduite. » (P. de Bourgoing, Souvenirs d'histoire contemporaine. Épisodes militaires et politiques, Paris, 1864, p. 571).

2. Vnešnjaja politika Rossii XIX - načala XX veka (La politique extérieure de la Russie auX $X I X^{\mathrm{e}}-$ début du $X X^{\mathrm{e}}$ siècles.), série $\mathrm{n}^{\circ} 1$, vol. 1-8; série $\mathrm{n}^{\circ} 2$, vol. 1-8 (16), Moscou, $1960-$ 1994. 
senta la France à Saint-Pétersbourg de 1835 à 1841, il fut le seul diplomate en poste en Russie à mériter une publication relativement complète ${ }^{3}$. Il faut ajouter que les dépêches des envoyés intéressent en premier lieu les historiens de la diplomatie, très sélectifs quant au choix du matériau de leurs recueils et de leurs articles. Ainsi les éditeurs de la Vnešnjaja politika Rossii ne sélectionnent pour leur échantillon que des rapports de diplomates qui se réfèrent à la situation internationale. Or, chaque ambassadeur était aussi obligé de décrire régulièrement les événements de politique intérieure de son pays de séjour. C'est dans ce genre de rapports, souvent mis à l'écart par les commentateurs, que l'on trouve non seulement de nouveaux faits importants, mais aussi des éléments permettant de revisiter, sous un angle inédit, des événements et des personnages très connus.

J'essaierai ci-dessous d'exposer la façon dont les diplomates français s'entretenaient avec l'empereur Nicolas Irr sur les sujets de politique intérieure française, ainsi que sa vision à lui de ces sujets, basée sur les renseignements fournis par son ambassadeur à Paris. Parmi un vaste corpus, ont été choisis deux épisodes qui eurent lieu en 1827 et en 1830. Le premier, à ma connaissance, semble être ignoré par les mémorialistes, tandis que le second, bien que fréquemment mentionné, peut être soumis, grâce aux matériaux d'archives, à une lecture nouvelle.

Les chroniqueurs ont déjà noté le fait que l'on suivait de près en Russie les affaires françaises lors de la Restauration. Ainsi le baron de Bourgoing, chargé d'affaires français à Saint-Pétersbourg en 1830, mentionné ci-dessus, écrit-il dans ses mémoires :

« On était habitué à Saint-Pétersbourg, depuis 1814 et 1815, à prendre une part très vive à tout ce qui se passait à Paris. La société de Saint-Pétersbourg, toujours très réservée dans ses discours, en ce qui concerne les questions russes de politique intérieure, s'exprimait souvent avec une certaine liberté sur celles touchant aux événements des autres pays. On y pouvait même distinguer, surtout à propos des affaires de France, des divisions et des nuances de parti, dont les dénominations étaient empruntées à notre langage parlementaire. »

Ayant souligné que Nicolas Ier, suivi par la majorité de ses notables, soutenait la droite centriste parisienne (donc, les adeptes de la monarchie constitutionnelle), selon une tradition datant de l'époque d'Alexandre Irer, Bourgoing continue :

« L'empereur Alexandre et son successeur toléraient cependant, même dans leur entourage, les sentiments et les discours inspirés par le parti que nous désignions en France par l'expression d'ultra. On peut même dire que cette couleur poli-

3. Cf. P. de Barante, Souvenirs, Paris, 1894-1901, vol. 1-8; voir également Donesenija francuzskih predstavitelej pri russkom dvore (Relations des envoyés français attachés à la cour russe), Saint-Pétersbourg, 1901-1908, vol.1-3 (Sbornik Imperatorskogo russkogo istoričeskogo obščestva, vol. 112, 119, 127) ; les relations publiées couvrent la période 18141820. 
tique ne déplaisait alors, sérieusement, à aucun souverain de l'Europe. Lorsque l'empereur Nicolas, en particulier, contredisait ou même tançait les adhérents de cette opinion, il ne le faisait guère qu'en souriant. Il n'aurait montré un front sévère qu'à ceux qui auraient exprimé des doctrines d'un libéralisme très prononcé, si de pareilles manifestations avaient pu se produire devant lui. $»^{4}$

De telles appréciations venant de la bouche de Nicolas Ier sont bien prévisibles. Cependant, les documents d'archives dont nous parlerons plus bas donnent une image moins triviale de l'empereur russe.

Depuis son intronisation, ayant eu lieu dans les circonstances catastrophiques que l'on connaît, Nicolas I ${ }^{\text {er }}$ ne cessa jamais de s'efforcer de convaincre les autres monarques européens, qui observaient la Russie non sans inquiétude, que les événements du 14 décembre étaient davantage fortuits que logiquement fondés, qu'ils n'avaient en rien corrompu le régime et que l'armée n'avait point soutenu les rebelles (ce dernier moment paraissait à l'empereur d'une importance cruciale, et il le soulignait constamment lors de ses conversations avec l'ambassadeur français, La Ferronnays). Ce qui demeure cependant moins connu, c'est que non seulement l'Europe était anxieuse à cause de la situation en Russie, mais que Nicolas luimême était alarmé par la situation en France. Et ceci non seulement par rapport à la révolution de Juillet, perçue naturellement par Nicolas avec beaucoup de méfiance, mais par rapport à la France de la deuxième moitié des années 1820, la France de Charles X. Les rapports de l'ambassadeur français La Ferronnays (conservés dans les archives du Quai d'Orsay) en apportent un précieux témoignage. (Il faut rappeler que Nicolas, à l'époque où il était grand-duc, connaissait déjà intimement La Ferronnays, le distinguant parmi les autres et étant donc plus ouvert avec lui qu'avec d'autres diplomates. La Ferronnays, dans ses rapports écrits après l'arrivée de Nicolas au pouvoir, le rappelle à maintes reprises ${ }^{5}$ ).

4. P. de Bourgoing, op. cit., p. 498-499. Bourgoing, au moment où il écrivait ces lignes, connaissait Nicolas depuis longtemps : il avait été présenté à ce dernier à Berlin en 1816, lorsque Nicolas n'était encore que grand-duc, et Bourgoing, deuxième secrétaire de l'ambassade de France. En relatant cet épisode, Bourgoing souligne la sympathie que Nicolas vouait à la France à cette époque : «Les premiers mots qu'il m'adressa [au bal] furent des paroles gracieuses pour mon pays et pour les souvenirs chers à mon patriotisme. Trop jeune, au temps des dernières guerres de l'Empire, pour y prendre part, il était resté étranger aux animosités, aux rancunes et aux préventions de cette époque. L'empereur Napoléon n'était pour lui qu'un personnage historique, un grand capitaine, digne de tout son enthousiasme. » (ibid., p. 417; sur la sympathie de Nicolas pour Napoléon, voir ibid.,p. 540-541). Voir également le témoignage du même Bourgoing démontrant qu'avant l'expédition algérienne de l'armée française de 1830, Nicolas ordonna à Černyšev, son ministre de la Guerre, de tirer des archives tous les matériaux utiles concernant la guerre contre la Turquie afin de les transmettre aux Français (ibid., p. 227-228). Sur les relations entre les cours russe et française, cf. S. S. Tatiščev, Imperator Nikolaj i inostrannye dvory (L'empereur Nicolas et les cours étrangères), Saint-Pétersbourg, 1889.

5. Cf. la dépêche de La Ferronnays du 15/27 décembre1825 : «Mes rapports personnels avec lui [le nouvel empereur] seront faciles et agréables ; il m'a constamment témoigné un intérêt assez vif pour donner, en ce moment, au corps diplomatique lieu de me féliciter de son avènement. J'allais souvent chez lui ; j'y suis quelquefois resté plusieurs heures, causant avec la plus grande familiarité. » (Archives du ministère des Affaires étrangères (cité infra AMAE), Correspondance politique. Russie, $\mathrm{n}^{\circ} 169$, f. 315). Le 1er janvier 1826 , le jeune empereur, ayant reçu tous les membres du corps diplomatique, invita ensuite La Ferronnays à passer dans son bureau 
La Ferronnays raconte dans sa dépêche du 4/16 janvier 1827 un entretien avec l'empereur, ayant eu lieu deux jours plus tôt dans le palais d'Hiver. Nicolas lui confie son inquiétude quant à « l'état intérieur de la France et la disposition des esprits » :

«C'est la première fois, depuis que l'Empereur est sur le trône, qu'il aborde cette question avec moi. "Vous savez, me dit Sa Majesté, que j'ai pour principe de ne point me mêler des affaires des autres, mais mon attachement pour le Roi et sa famille, mon intérêt pour le bonheur de la France ne me permettent pas de rester indifférent aux dangers qui peuvent les menacer. Je vous avoue que j'ai reçu des informations auxquelles j'ajoute une grande confiance, qui me font craindre que l'esprit public ne soit plus aussi bon qu'il l'était. Ce sont surtout les renseignements qui me parviennent sur les dispositions de l'armée qui me causent de vives inquiétudes. On assure qu'il existe un grand découragement parmi les officiers même de la garde, que les soldats sont mécontents et dégoûtés du service au point d'avoir exprimé hautement leurs regrets sur les apparences d'une guerre avec l'Angleterre. Cette disposition est trop contraire à l'esprit militaire de toutes les nations et surtout des Français pour ne pas en être surpris et n'en pas concevoir quelques alarmes. Ce n'est point ici l'Empereur de Russie qui se permet de faire ces observations à l'Ambassadeur de France, c'est un ami sincère du Roi qui exprime franchement des inquiétudes qui lui paraissent fondées. Dans ce moment, la sûreté du trône, celle de la France, notre tranquillité à tous, reposent, en quelque sorte, sur l'armée française. Si son dévouement au trône est douteux, si son esprit militaire est éteint, il est permis de tout craindre et de ne regarder l'avenir qu'avec effroi. [...] J'ai des preuves certaines qu'il existe dans la plupart des officiers de l'armée un grand dégoût pour le service, que ce dégoût gagne le soldat qui n'a pas assez de considération pour ses chefs." »

Ce à quoi La Ferronnays objecte que ces peurs sont peu fondées. Certes, il existe des personnes parmi les hommes d'épée perpétuellement «chagrines et frondeuses », qui aspirent à la guerre en temps de paix et à la paix en temps de guerre. Or l'armée française, lorsqu'il est question d'action, a toujours su se montrer digne de sa réputation, comme en témoigne la guerre d'Espagne de 1823.

et causa plus d'une heure avec lui en tête-à-tête. Il commença cet entretien en disant : «Combien je suis heureux de me trouver avec vous et de pouvoir une fois librement épancher mon cœur dans celui d'un ami qui saura me comprendre!» (P. Wakar, «Les rapports de l'ambassade de France à Saint-Pétersbourg sur la conjuration des décembristes », Le Monde slave, 12, 1925, p. 450-451), et l'acheva sur: «Ce que je sais, c'est que tant que vous serez avec nous, je croirai à l'amitié du Roi pour moi et que toute affaire quelconque sera toujours facile de traiter entre nous deux. Promettez-moi, mon cher comte, de ne plus faire de démarches pour nous quitter. Je le demande à votre amitié. » (AMAE, Correspondance politique. Russie, $\mathrm{n}^{\circ} 170$, f. 24 v.-25). Cf. également le témoignage de Bourgoing : lorsque celui-ci tâche de convaincre l'empereur de ne pas déclarer la guerre à la France de la révolution de Juillet, il argumente que « les Français que vous estimez le plus, le duc de Mortemart et le comte de La Ferronnays, vous le diraient comme moi », et Nicolas de dire : «Oui, je sais qu'en vous écoutant, c'est l'opinion de la France modérée que j'interroge, et que c'est comme si La Ferronnays ou Mortemart me parlaient. » (P. de Bourgoing, op. cit., p. 516). 


\section{L’empereur défend néanmoins sa position :}

«Vos libéraux auront beau dire, ce sont les armées fortes, disciplinées et dévouées qui assurent les trônes, qui maintiennent la tranquillité au-dedans et qui surtout font respecter les États au dehors. Ils disent de belles choses sur les institutions anglaises ; je suis le premier à les admirer, mais ôtez à l'Angleterre sa force maritime, et vous verrez comment l'Europe en agira avec elle. [...] Du reste, ceci est une conversation entre deux hommes francs et loyaux. Cet entretien ne peut avoir aucun caractère officiel. ${ }^{6}$

Un mois plus tard Nicolas revient dans une autre conversation avec La Ferronnays sur la situation intérieure de la France. L'ambassadeur communique les résultats de cet entretien dans sa relation du 2 février 1827 adressée au baron de Damas, ministre des Affaires étrangères. Vienne, écrit La Ferronnays, persévère à encourager l'inquiétude de l'empereur «par des moyens trop connus $»^{7}$ :

«Depuis quelque temps les lettres particulières et même officielles adressées à Sa Majesté de plusieurs parties de l'Italie et de la Suisse, semblent annoncer une

6. AMAE, Correspondance politique. Russie, $n^{\circ} 172$, f. $40-41$ v., 44-44 v. Cette croyance dans l'armée comme fondement de l'État explique la persistance avec laquelle Nicolas, aussitôt après son intronisation, souligne devant tous les envoyés étrangers, comme il le fait devant La Ferronnays, le fait que seule une poignée d'officiers participa à la révolte du 14 décembre. La partie principale de l'armée serait restée fidèle à Nicolas, proche du corps militaire.

7. Deux ans plus tard, l'ambassadeur russe à Paris, le comte Pozzo di Borgo, stigmatisa avec emphase les tentatives de Metternich de présenter la France comme la source de tous les maux politiques du monde (relation du 15/27 novembre 1829) : «Le Prince de Metternich, déjoué et humilié par les résultats de la guerre contre la Turquie, cherche une idée générale dont il travaille à se faire l'auteur et le chef pour se reprendre la présidence du système qu'il voudrait former et cette idée a pour but d'appeler tous les souverains à se prémunir contre les révolutions prochaines dont, selon lui, le foyer est en France. Sans doute, il existe dans le monde et en France des germes de révolution ; mais lui qui veut être le premier à les combattre, pourquoi donne-t-il au Duc de Reichstadt une si grande et si éclatante existence ? Pourquoi l'effigie de ce fantôme estelle répandue sur la toile et sur le papier par des agents que la police française a plusieurs fois découvert provenir de l'Autriche elle-même ? On dirait qu'il a besoin d'agitation dans ce pays pour appeler les autres puissances à les comprimer. Tantôt il encourage les ultraroyalistes les plus effrénés, et avec eux les prêtres et les Jésuites, et tantôt les Bonapartistes et les ennemis des Bourbons. » (Arhiv vnešnej politiki Rossijskoj Imperii - AVPRI, f. Kanceljarija, op. 468, d. 9167,1.322-322 ob.). Metternich était guidé dans cette attitude non seulement par sa méfiance foncière vis-à-vis d'une France constitutionnelle et beaucoup trop libérale à son gré, mais surtout par l'envie de la pousser à se quereller avec ses alliés, Russie en tête, à propos de la question d'Orient (cf. A. Debidur, Diplomatičeskaja istorija Evropy (L'histoire diplomatique de l'Europe), Rostov-sur-le-Don, 1995, vol. 1, p. 246-249). Voir également l'instruction communiquée, le 17 janvier 1829, par Metternich à l'ambassadeur austro-hongrois à Saint-Pétersbourg, le comte de Ficquelmont, qui montre bien son opinion sur les relations franco-russes, de même que sur la personnalité de La Ferronnays, devenu déjà à cette époque ministre des Affaires étrangères (il le fut du 4 janvier 1828 au 14 mai 1829) : « De bien malheureuses relations n'ont depuis longtemps cessé d'exister entre la France et la Russie. Je qualifie ces relations de malheureuses, parce qu'elles n'ont jamais porté que sur le soutien de principes fallacieux ou d'entreprises menaçantes pour le repos de l'Europe. Ce qui augmente ce danger, ce sont les graves erreurs auxquelles un grand nombre de royalistes français se sont livrés. Dupes de leurs fausses théories de gloire nationale, et en même temps des ruses des révolutionnaires, ces hommes caressent les projets les plus aventureux, et leur tendance si prononcée vers la prépondérance russe n'a d'autre base que l'espoir de pouvoir servir également, par cette prépondérance, les intérêts de la puissance française. Nous avons vu pendant de longues années le Cabinet de Saint-Pétersbourg 
disposition prochaine à de nouvelles agitations. Les révolutionnaires, quelle que soit la dénomination qu'on leur donne, ne seraient plus, d'après ces lettres, les premiers moteurs des troubles qu'on présage, mais ils se tiendraient prêts à profiter des fautes de leurs adversaires et à faire tourner à leur avantage les armes mêmes et les moyens que l'on aurait voulu employer pour les combattre. C'est de l'exagération du bien que l'on prétend que le mal peut éclore, c'est d'une tendance trop précipitée vers l'intolérance religieuse et qui enflamme les passions de ceux qui s'y laissent entraîner comme elle irrite ceux qui lui résistent, que l'on craint de voir naître le désordre. Le zèle impatient qui veut arriver à son but sans considérer que la précipitation détruit presque toujours l'effet que l'on veut produire ; ce zèle dur et aveugle qui pour réformer brusquement quelques abus, heurte sans prudence et sans égards les intérêts, les opinions coupables ou légitimes, enfin ce zèle indiscret et sans lumières qui substitue des écarts à ceux qu'il attaque, voilà ce que l'on signale comme cause première des nouvelles agitations que l'on prédit. L'Empereur m'en a parlé avec une chaleur qui m'a étonné. - C'est un sujet fort délicat à traiter, surtout avec un Prince d'une croyance différente. Sa Majesté m'a demandé si je faisais partie de la Congrégation ; je lui ai répondu que je ne la connaissais que par ce que j'en entendais dire et ce que je lisais dans les gazettes ; que je croyais que ce mot de Congrégation était, comme celui de féodalité 8 , un épouvantail imaginaire dont se servaient avec succès les gens mal intentionnés pour égarer les esprits et irriter les passions ; que je ne croyais ni à l'existence de la Congrégation, telle qu'on la dépeignait, ni à la puissance qu'on lui supposait ; que dans tous les cas, j'avais en horreur toutes associations secrètes, quel que pût en être le but ; que je les regardais comme une puissance dans l'Etat, à laquelle aucun sujet fidèle, aucun homme loyal ne pouvait se rallier et que je ne serais pas plus disposé à me faire congréganiste que carbonaro. L'Empereur m'a paru singulièrement préoccupé de l'existence prétendue de cette association et du mal qu'elle pouvait faire ; ce qui m'a prouvé que les moyens qu'on emploie pour frapper son imagination de la possibilité de quelque nouvelle catastrophe, produisent quelque effet. Il ne serait peut-être pas inutile, Monsieur le Baron, de me mettre en mesure de pouvoir une autre fois répondre avec une plus grande connaissance de cause à l'Empereur, car il m'est facile de prévoir que c'est un sujet sur lequel il ramènera souvent la conversation. Je lui ai dit la vérité, - je ne suis ni ne serai jamais de la Congrégation ; je ne crois point à son existence ; mais si elle existe, je crois qu'elle ne peut être que funeste et dangereuse. Vous voyez, Monsieur le Baron, que j'ai moi-même besoin d'être éclairé sur cette question qui devient un moyen d'alarmer d'autres gouvernements sur la stabilité de la France. »9

se livrer à un système de ménagements extraordinaires pour les idées libérales, et suivre même ce système à des époques où l'Empereur Alexandre était devenu l'adversaire décidé de l'esprit révolutionnaire. C'est à ces époques que l'influence du général Pozzo était prépondérante dans le conseil tout libéral de Louis XVIII. [ ... C'est ainsi que nous avons vu M. de Chateaubriand arborer, sous l'égide de ce même ambassadeur, à la fois les couleurs révolutionnaires et russes. M. le comte de La Ferronnays, qui, sans contredit, est aussi Russe que Français, fait en même temps partie du plus libéral des ministères que la France ait encore eus. [...] Un ministère aussi faible que celui qui gouverne aujourd'hui la France ne saurait compter comme un contrepoids dans la balance du mal. Sa tendance est décidément moins monarchique qu'elle n'est révolutionnaire et russe. » (Prince de Metternich, Mémoires, documents et écrits divers, Paris, 1881, vol. 4, p. 576-577)

8. La Ferronnays parle ici de la mode libérale qui consiste à utiliser les termes de « féodalité » et de « féodaux » pour désigner tout courant conservateur, rétrograde ou dirigé vers la restriction de la liberté politique.

9. AMAE, Correspondance politique. Russie, $\mathrm{n}^{\circ} 172$, f. $78-80 \mathrm{v}$. 
Le discours de l'empereur, tel que le retranscrit La Ferronnays, est bien alambiqué. Pourtant les faits cachés derrière ces belles et pompeuses paroles sont plus que concrets : Nicolas Ier, connu comme conservateur et réactionnaire, se prononce ici contre les courants ultra-royalistes, ultra-conservateurs ou ultra-catholiques de la société française. Ce faisant, il n'agit pas ainsi parce que ces courants lui sont particulièrement antipathiques en soi, mais parce qu'ils risquent de briser l'équilibre politique existant en France. Mieux encore, l'empereur russe semble suivre la situation intérieure de la France si attentivement qu'il analyse non seulement des événements factuels, mais aussi leur miroitement «mythologique » dans la conscience collective des Français. Ainsi, il interroge La Ferronnays sur la Congrégation, qu'il connaît apparemment de plus près que l'ambassadeur français, qui, lui, réclame des renseignements et des commentaires sur le sujet auprès de son ministre.

Il n'y a pas lieu d'exposer en détail la question de la Congrégation dans le cadre de cette étude ${ }^{10}$. Il suffit de dire que sous l'influence des écrits libéraux (comme ceux du journal Le Constitutionnel au cours des années 1824-1826, ainsi que des textes des penseurs «gallicans », qui s'opposaient aux jésuites), une idée reçue s'empara de la conscience collective française. La croyance se répandait que la politique de la France, intérieure comme extérieure, était dirigée et définie non pas par les organes légitimes (que ce soit le conseil des ministres ou la chambre des députés), mais par la Congrégation, organisation secrète ultra-catholique plaçant partout ses hommes et imposant peu à peu son ordre à la société. Cette légende semblait d'autant plus véridique qu'une vraie Congrégation existait en France vers cette époque. Elle fut fondée en 1801 par le père Delpuits, et fut dirigée pendant la Restauration par le père Ronsin. Comme toutes les autres congrégations catholiques, celle-ci était vouée à la Vierge Marie. Ses membres s'occupaient essentiellement d'œuvres de charité et ne possédaient aucunement l'influence politique que leur attribuaient les sources libérales ${ }^{11}$. Cette influence pouvait en revanche être effectivement exercée par une autre organisation, celle-là bien secrète et politisée. Il s'agit de l'association des « Chevaliers de la Foi », fondée en 1810 par Ferdinand de Bertier de Sauvigny et construite selon le modèle des loges maçonniques, qui avait pour but de restaurer la monarchie légitime.

Il est incontestable que les deux organisations étaient plus ou moins liées: certains « chevaliers » (mais on est loin de la totalité des membres) faisaient partie de la Congrégation et vice-versa. De même, il faut admettre que, lors des dernières années du règne de Louis XVIII et surtout à l'époque de Charles X, l'influence du clergé sur la politique du royaume se renforça considérablement. D'un autre côté, il

10. Pour l'histoire détaillée de ce «mythe », voir G. de Bertier de Sauvigny, Le comte Ferdinand de Bertier et l'énigme de la Congrégation, Paris, 1948 ; cf. id., La Restauration, Paris, 1955, p. 381-386 ; E. de Waresquiel, B. Yvert, Histoire de la Restauration, 1814-1830. Naissance de la France moderne, Paris, 1996, p. 384-386 ; M. Leroy, Le mythe jésuite, Paris, 1992, passim (trad. russe : Mif o iezuitah, Moscou, 2001).

11. Le questionnement de La Ferronnays concernant la Congrégation, adressé au ministre des Affaires étrangères, le baron de Damas, est d'autant plus piquant que Damas lui-même en fut membre assidu. 
est tout aussi incontestable que les journalistes libéraux, ayant transformé le fait réel, celui de la connivence entre Charles $\mathrm{X}$ et les représentants de la droite cléricale et conservatrice en un mythe de la Congrégation toute-puissante, en exagérèrent la force. En effet, au moment où tous les journalistes libéraux concentraient leurs écrits sur la menace de la Congrégation, l'association des «Chevaliers de la Foi », qui était visée, s'arrêta d'exister (elle fut dissoute selon le vœu de son grand maître, Mathieu de Montmorency) ${ }^{12}$. Les journaux continuaient néanmoins à révéler «le danger ecclésiastique » provenant de la Congrégation. C'est surtout dans la presse de l'époque que quiconque intéressé par la situation intérieure de la France pouvait apprendre que le conseil assigné par le roi n'était qu'un pantin entre les mains d'un vrai conseil secret et percevoir dans cet état des choses une menace pour la stabilité du pays.

En théorie, il n'est pas improbable que l'empereur russe ait pu faire partie des lecteurs de ces mêmes journaux. Il est cependant plus plausible qu'il ait puisé ses renseignements sur la Congrégation par le biais d'un autre canal, celui des relations de son ambassadeur à Paris, Karl Osipovič (Charles André) Pozzo di Borgo (17641842). Lorsque La Ferronnays estimait que l'auguste inquiétude concernant l'armée française (qu'il jugeait d'ailleurs suscitée par des informations erronées) provenait d'une source autre que les rapports de $\mathrm{Pozzo}^{13}$, il avait entièrement raison car la question de l'armée n'était quasiment pas mentionnée dans les dépêches de Pozzo datant de 1826 (début 1827). Quant au fait que La Ferronnays passa sous silence les origines des informations parvenues à Nicolas Ier sur la Congrégation et sur son influence néfaste, c'est que de telles informations ne pouvaient de toute évidence venir d'ailleurs que de Pozzo di Borgo. En effet, Pozzo, dans toutes ses relations, ne parlait que de la politique erronée de Charles X, qui, s'entourant de plus en plus d'ultra-catholiques, s'opposait ainsi non seulement aux libéraux fanatiques, mais à la société française dans son ensemble ${ }^{14}$.

12. Le biographe du roi Charles $X$ décrit très bien la situation: «La véritable organisation secrète, hiérarchisée, politisée, la milice de combat, ce ne fut pas la Congrégation dont on parlait tant, ce furent les Chevaliers de la Foi dont on ne parla jamais. » (J. Cabanis, Charles X roi ultra, Paris, 1972, p. 264). Le roman de Stendal, Le rouge et le noir (pt. II, chap. 23), montre bien à quel point la Congrégation était « mythifiée » : lorsque les conspirateurs ultra-royalistes envoient Julien Sorel à l'étranger afin de faire parvenir aux alliés leur «note secrète », il est espionné par un membre de la Congrégation qui, selon toute probabilité historique, aurait dû seconder le complot royaliste. Ainsi la Congrégation et ses membres personnifiaient le Mal même à l'encontre du sens et de la logique historiques.

13. Selon La Ferronnays, les nouvelles de la situation précaire de l'armée française pouvaient provenir du prince Nikolaj Andreevič Dolgorukij (1792-1847), envoyé à Paris en 1826 (AMAE, Correspondance politique. Russie, $\mathrm{n}^{\circ}$ 172, f. 44 v.) ; cf. la relation de Pozzo di Borgo du 23 mai/5 juin 1826 (AVPRI, f. Kanceljarija, op. 468, d. 9148,1. 188).

14. Les relations de Pozzo di Borgo, qui constituent une source historique précieuse, ne sont encore que très partiellement publiées. Il fut ambassadeur à Paris pendant vingt ans, de 1814 à 1834. À part les relations choisies incluses dans Vnešnjaja politika Rossii, mentionnée ci-dessus, seule sa correspondance diplomatique de 1814 à 1818 vit le jour (Ch.-A. Pozzo di Borgo, Correspondance diplomatique, 1814-1818, Paris, 1890-1897, vol. 1-2). 
Pozzo rédige de la façon suivante son rapport du 28 mars/ 9 avril 1826 à l'attention du vice-chancelier russe K. V. Nesselrode :

«Zélés pour la propagation de bonnes doctrines politiques et pour la religion de l'Etat, les hommes qui se sont réunis dans ce but et qu'on désigne sous le nom de Congrégation, redoublent d'activité pour inculquer ce qu'ils regardent comme nécessaire et pour dénoncer ce qu'ils envisagent comme dangereux. [...] [ils] forment, en quelque sorte, une puissance dans l'État [...] Le Roi, sans les avouer, est censé les protéger, soit parce qu'en général ces individus sont des personnes qui lui ont montré du dévouement, soit parce que Sa Majesté est persuadée qu'Elle n'a pas de sujets plus fidèles. Parmi les moyens que cette classe de Royalistes regarde comme les plus propres à consolider le trône légitime et à purger la société de la corruption qui la travaille est le rétablissement des Jésuites ${ }^{15}$. [...] Sans vouloir scruter les avantages ou les inconvénients de cette puissance latérale qui s'est élevée à côté de la puissance publique, dans le but de la soutenir, la vérité est que son apparition et son développement excitent l'animadversion du parti qui la redoute sincèrement ou qui en fait un prétexte pour alarmer le peuple. La magistrature, les hommes d'une opinion modérée et la nation en général en tant qu'il est permis d'en interpréter les sentiments, appartiennent à la première classe. Les libéraux, les doctrinaires, les personnes mécontentes du gouvernement, les révolutionnaires enfin forment la seconde ; malheureusement toutes les deux s'accordent à déclamer contre une innovation qui les blesse pour des motifs différents, et ils la présentent au public comme une invasion dangereuse qui tend à asservir son esprit et à violer ses droits. Le ministère est lui-même divisé jusqu'à un certain point sur cet objet. MM. De Damas, de Clermont-Tonnerre, l'Evêque d'Hermopolis et, quoiqu'en sous-ordre, le directeur des postes et celui de la police, favorisent le clergé et les Jésuites à outrance ; M. de Villèle, dans le fond, est indifférent à tout, mais il est obligé de se conduire avec ménagement envers une classe qui forme une grande partie des Royalistes et qu'il ne saurait mécontenter sans s'exposer à perdre sa place. Ces divisions, M. le Comte, sont le germe de toutes les controverses dont l'opinion publique est agitée par les journaux et qui occupent souvent l'attention des tribunaux. Tantôt c'est contre une production impie qu'on s'élève à juste titre, et tantôt contre une doctrine sur l'étendue de la suprématie du Pape. Personne n'aurait imaginé que la France s'occuperait et serait même inquiétée à l'époque où nous sommes par ces sortes de discussions. Ceux qui les ont mises à l'avant, ont commis, à mon avis, une grande faute dont toutes les conséquences ne sont pas suffisamment appréciées jusqu'aujourd'hui. Je crains que la cour et le clergé n'aient voulu trop entreprendre et que leur influence, si nécessaire à conserver, ne diminue auprès de la masse tandis qu'elle croit faire des conquêtes en agissant sur les individus. »16

15. L'ordre des jésuites fut liquidé en Europe en 1773 par la bulle du pape Clément XIV. Les jésuites revinrent en France juste après la Restauration. Toutefois leur existence manquait de légitimité. Ils constituaient, unis à la terrible Congrégation, la cible préférée de la presse libérale (et surtout du jounal Le Constitutionnel) qui, exagérant beaucoup leur influence sur la vie politique du pays, voyait en eux l'ennemi principal de la nouvelle France.

16. AVPRI,f. Kanceljarija, op. 468, d.9147,1.315-317 ob. ; texte rédigé en français. Sont mentionnés dans ce rapport : Jean Baptiste Guillaume Joseph, comte de Villèle ( 1773-1854), président du Conseil de 1822 à 1827 (sur les difficultés de sa position liées à sa dépendance de différents partis politiques contemporains voir: J.Cabanis, op. cit.,p. 262-287); Maxence, baron de Damas (1785-1862), ministre des Affaires étrangères de 1824 à 1828 ; Aimé Marie Gaspard, duc de Clermont-Tonnerre (1779-1865), ministre de la Guerre de 1824 à 1828, et Denis Antoine Luc, comte de Frayssinous (1765-1841), évêque d'Hermopolis, ministre des Affaires ecclésiastiques et de l'Instruction publique de 1824 à 1828 . 
Le 16/28 avril 1826, Pozzo nota, concernant l'intention affichée du roi de « faire un sacrifice expiatoire sur la place Louis XV, à l'endroit même où Louis XVI perdit la vie » : « Il y a de l'imprudence à exiger du peuple corrompu et léger de cette capitale des sentiments qu'il n'a pas et des démonstrations qu'il refuse. »17

Le 10/22 décembre 1826 (presque à la veille de la conversation entre Nicolas Ier et La Ferronnays que nous commentons), Pozzo conclut ainsi ses observations :

«La France est forte du nombre de ses habitants, de la contiguïté de son territoire, de ses ressources et j'ajouterai de ses institutions, surtout si elles étaient exécutées et entendues dans un esprit de bonne foi d'un côté et de modération de l'autre. Ces avantages sont contrebalancés à la vérité, et même affaiblis par une sorte d'inefficacité, si j'ose m'exprimer ainsi, dans laquelle le Roi se laisse choir insensiblement par l'encouragement donné au zèle inconsidéré d'un clergé des hommes désignés sous le nom des congréganistes, qui allient l'ambition à une sorte de piété affectée et qui compromettent la personne du Roi, censé les favoriser en secret [...]. »18

Au lieu de proposer une loi draconienne sur la presse, le gouvernement français aurait dû, d'après Pozzo, « gouverner avec une grande supériorité de vues, à ne pas déchoir par des défauts de caractère, par des faussetés et des puérilités habituelles dans la considération du public, à ne pas contracter l'esprit de secte qui n'est pas celui de la religion, à s'abstenir de traiter la France comme si elle était païenne ou idolâtre [...]. »19

Enfin, le 13/ 25 janvier 1827, Pozzo résuma l'ensemble de la situation en France dans un rapport adressé à Nesselrode, son chef direct - et par procuration à l'empereur Nicolas en personne. Il explique dans son texte que Charles X désire concilier «les institutions octroyées par son frère », à savoir les mécanismes du gouvernement constitutionnel, avec le soutien secret des «personnes de sa prédilection », capables de « porter la France vers les idées religieuses et monarchiques que le ministère du duc de Richelieu ne lui semblait pas assez protéger », et que ce dualisme crée un schisme profond aussi bien au sein du cabinet que dans la société. Les ministres, souligne Pozzo, ayant observé les sympathies du souverain, se firent membres de la Congrégation. Pis encore, le premier ministre, Villèle, dut leur céder, provoquant ainsi la colère et le mécontentement du pays entier. «De tous ces éléments de faiblesse, de duplicité et de confusion est sorti un cri universel de réprobation, agrandi par l'animosité des partis opposés et répété par les talents des écrivains les plus distingués de la capitale et du Royaume. ${ }^{20}$ Ainsi :

« Dès que le Roi s'est fait, pour ainsi dire, chef de parti, dès qu'il regarde le clergé comme son premier appui et qu'il en fait un cas de conscience ; dès qu'il

17. Ibid., $1.399 \mathrm{ob.}$

18. Ibid., d. $9152,1.478$ ob.-479; texte rédigé en français.

19. Ibid., 1.481 ob.

20. Ibid., d. 9154,1. 124 ob.-127 ; texte rédigé en français. 
repousse enfin les hommes qui n'entrent pas dans ses idées personnelles, alors la formation d'un nouveau ministère lui devient impossible, s'il doit être plus populaire, et dangereuse, s'il le choisit parmi des gens plus odieux que ceux qui administrent maintenant [car les adversaires de l'ordre actuel ne font qu'attendre des circonstances extraordinaires qui leur seraient profitables] au détriment du Roi et de sa famille.»

Pozzo conclut d'ailleurs que, même si le danger est sans aucun doute présent, il n'est pas encore imminent : la prospérité matérielle des Français est encore trop importante, et leur haine envers le «parti religieux » n'est pas suffisamment aiguë pour provoquer des confrontations ouvertes ${ }^{21}$.

La position de Pozzo est claire : il considère les tendances de Charles $\mathrm{X}$ à s'appuyer (en contournant la constitution) sur les milieux cléricaux comme un facteur néfaste, qui contient en outre la menace sous-jacente de diviser la population, de déstabiliser l'État et de donner aux libéraux des motifs de critiquer - avec justesse - le gouvernement ${ }^{22}$. Ce fut précisément ce raisonnement que reproduisit l'empereur russe dans son entretien avec l'ambassadeur de France. Le monarque se montra non seulement au courant de la situation «franco-française », mais il sut également (et ceci grâce à l'information fournie par Pozzo) évaluer d'une façon adéquate les dangers et les risques d'une telle situation. Qui plus est, il n'hésita pas à condamner les actions des forces politiques conservatrices qui, d'après ce que l'on sait communément sur ses convictions politiques personnelles, auraient dû, au contraire, susciter toute sa sympathie.

Le même sujet - « l'empereur Nicolas défend la constitution française des violations infligées par le roi Charles $\mathrm{X} »-$ transparaît d'une manière encore plus flagrante dans les dépêches envoyées depuis Saint-Pétersbourg vers la France en 1830. Au lieu de La Ferronnays, rapatrié, un autre diplomate en est l'auteur: Casimir Louis Victorien de Rochechouart, duc de Mortemart (1787-1875), ambassadeur de 1829 à 1831. Mortemart jouit, comme jadis La Ferronnays, de la disposition particulièrement amicale de l'empereur envers lui. Ainsi, lorsqu'il revint en Russie en 1829 après son congé à Paris et à Vienne, l'empereur l'appela « camarade de camp » (sous-entendant la présence de Mortemart dans l'état-major de l'armée russe, lors de la récente guerre contre la Turquie) et « le conduisit chez

21. Ibid.,1. 131-131 ob.

22. On trouve un écho à ce point de vue dans une lettre privée russe (A. Ja. Bulgakov à son frère, le 2 août 1830, avant l'arrivée des premières nouvelles de la Révolution) : « Les Français m'insupportent ! Regarde le Roi, il a tout et il n'est jamais content de rien ! Je ne peux sentir ces dévots qui ne parlent que de l'office et de la pudeur, tout en semant la guerre et les désaccords. Je crains un tohu-bohu, car la situation du Roi semble critique. Qu'il puisse au moins sacrifier ses ministres afin de se sécuriser et de calmer le peuple! Alors, il continuera à régner, mais dépourvu à tout jamais de l'amour et du respect de ses sujets. » (Russkij arhiv, 3, 1901, p. 493). 
l'Impératrice et ses enfants comme un simple particulier »23. En cette année 1830, tout comme en 1827, l'empereur tire ses informations, voire ses parti pris en matière d'affaires françaises, des relations de Pozzo, qui reste toujours ambassadeur à Paris.

Si dans les années 1826-1827, malgré ses sinistres prévisions sur les séquelles possibles de la politique de Charles X, Pozzo ne trouvait pas la situation française désespérée, son attitude changea à partir de la fin de 1829. Le 8 août 1829 eut lieu un changement de ministère. Charles $\mathrm{X}$ arriva enfin à mettre en œuvre son rêve ultime, celui d'avoir à la tête de son cabinet un homme qui partageât ses opinions ultra-conservatrices. Cet homme était le prince de Polignac (tout d'abord il ne fut que ministre des Affaires étrangères et ne devint président du Conseil que le 17 novembre 1829 , mais c'est incontestablement lui que l'opinion publique croyait être responsable de l'idéologie du nouveau cabinet). Or, Pozzo avait écrit avant l'arrivée de Polignac à la tête du gouvernement que son avènement serait une chose fort peu souhaitable.

La société, selon Pozzo, récusait Polignac et sa politique. Le roi pouvait imposer à la société une politique impopulaire, mais au prix d'une discordance contenant des dangers qui « surpassent tous les calculs ». « Ceux qui ne savent pas les concevoir aujourd'hui, parce qu'ils ne sont pas imminents et qu'il faut attendre quatre mois avant de les voir en action, seront les premiers à en être consternés et à se livrer probablement à des personnes dont les exigences seront sévères et peut-être fatales. $\gg^{24}$ L'attitude de Pozzo envers Polignac était également conditionnée par la politique internationale : Polignac, ambassadeur français en Angleterre jusqu'à 1829, soutenait souvent les Britanniques contre les Russes (par exemple sur la question d'Orient), ce qui devait naturellement agacer l'envoyé russe. Pozzo partageait l'idée, commune en France, selon laquelle Polignac n'était pas une figure politique autonome, mais le protégé et l'agent du premier ministre britannique, le duc de Wellington ${ }^{25}$. Pourtant l'effarement de Pozzo à la nouvelle de l'installation de l'ancien ambassadeur dans le fauteuil du ministre des affaires étrangères ne reposait pas seulement sur ces causes extérieures. Pozzo voyait clairement « les sentiments de mépris et d'indignation » après la création du « ministère antinational ${ }^{26}$, ainsi que les conséquences d'une telle action, à savoir une nouvelle alliance réunissant la droite

23. P. de Bourgoing, Le duc de Mortemart et le baron de Bourgoing. Souvenirs anecdotiques, Paris, 1904, p. 125 (voir la lettre de Mortemart à Portalis, ministre des Affaires étrangères, sans date).

24. AVPRI, f. Kanceljarija, op. 468, d. 9166,1.231-232 (relation du 25 juillet/6 août 1829 citée en partie in F. F. Martens, Sobranie traktov i konvencij zaključennyh Rossieju s inostrannymi deržavami (Recueil des traités et des conventions conclues entre la Russie et les puissances étrangères), Saint-Pétersbourg, 1909, vol. 15, p. 79).

25. F. F. Martens, op. cit., vol. 15, p. 71, 74 sq. Pozzo écrit dans sa relation du 5/17 août 1829 qu'au cours des deux dernières années, «à chaque période où le besoin du changement du ministère lui devenait sensible », Wellington « détachait » Polignac, l'envoyant aussitôt à Paris afin qu'il fût plus près des événements et qu'il ne ratât pas le portefeuille ministériel. (AVPRI, f. Kanceljarija, op. 468, d.9166,1.265 ob.).

26. AVPRI, f. Kanceljarija, op. 468, d. 9166,1. 267 (relation du 5/17 août 1829). 
modérée qui refusait la coopération avec Polignac, et la gauche. Pozzo considérait le nouveau gouvernement comme inutile et néfaste pour le roi même : «Le Roi n'a obtenu par l'espèce de coup d'État qu'on lui a fait commettre et auquel il était naturellement enclin et par le mode dont il y a procédé, que la satisfaction de devenir plus maître des hommes qui composent son conseil et plus faible envers sa nation. $»^{27} \mathrm{En}$ observant les changements funestes de l'opinion publique après la nomination de Polignac, Pozzo conclut que la propagande de la presse n'en fut pas la cause, mais uniquement un facteur aggravant : ainsi, Pozzo constatait que, à part La Quotidienne et la Gazette de France, tous les journaux parisiens frondaient le régime : ils « dissèquent et avilissent le nouveau ministère en masse et en détail avec une fureur et souvent une habileté qui emporte tout et qui compromet sinon l'existence, du moins le repos et la dignité du Roi lui-même », d'autant plus facilement que « lorsque les écrivains et les gens de lettres dont Paris et la France sont couverts cherchent à exciter des sentiments que le public ne partage pas, ou qu'ils veulent accréditer des faits contraires à l'évidence, ils ne sont qu'incommodes, mais dès que leurs productions se rencontrent avec les dispositions de la grande majorité des pays et qu'ils lui indiquent avec art des choses qui causent déjà des appréhensions, leur influence sur les esprits est incommensurable. ${ }^{28}$ De là, Pozzo entrevoyait le scénario fatal : « La prétention de gouverner la France par ordonnances est insensée. Le Roi et la dynastie passeraient des convulsions à la ruine. $»^{29}$ Le 25 août/6 septembre 1829, Pozzo émettait un pronostic encore plus détaillé : si le conseil présidé par Polignac ne trouve pas de soutien auprès de la chambre des députés, et si le roi est obligé de nommer un nouveau gouvernement, contraint à son tour d'être défendu contre la malveillance des élus, « il ne lui restera d'autre parti à prendre que d'abolir les lois existantes par des ordonnances et de se donner des députés de son propre choix. Cette mesure mettra la dynastie en péril et personne au monde n'oserait prescrire le terme de la réaction qu'elle risquerait de faire naître. $»^{30}$

27. Ibid., d. $9167,1.312$ (relation du 15/27 novembre 1829).

28. Ibid., d. $9166,1.267$ ob.-268.

29. Ibid., 1. 283 (cf. F. F. Martens, op. cit., vol. 15, p. 81).

30. AVPRI, f. Kanceljarija, op. 468, d. 9167, 1. 44 ob. Il est intéressant de noter que, d'après l'ambassadeur de Louis-Philippe en Russie, le général Athalin, Charles X induisit sciemment en erreur l'ambassadeur russe, lui cachant les mesures soigneusement préparées et restées secrètes (personne n'avait été initié au secret, hormis les membres du conseil des ministres). « Il paraît impossible - écrit Athalin dans ses "Notes", datées du septembre 1830 - que Sa Majesté l'Empereur [...] ne reconnaisse pas qu'il y a eu déloyauté insigne dans les actes qui ont amené la catastrophe de Charles X, lorsque lui-même paraît avoir eu fortement à se plaindre de la duplicité avec laquelle le roi en agissait envers son ambassadeur à Paris. Le soin que le Roi Charles X prenait de l'assurer, peu de jours avant la publication des ordonnances, qu'il était loin de sa pensée de rien entreprendre qui fût contraire à la foi jurée, prouve à la fois qu'il sentait toute l'importance du pas qu'il allait faire, et que c'était l'exécution d'un plan depuis longtemps médité et arrêté, mais pour laquelle il ne pourrait compter sur aucun appui de la part de la Russie dont un pareil attentat révolterait la loyauté. » (AMAE, Correspondance politique. Russie, $n^{\circ} 181$, vol. 11). Cette tromperie intentionnelle devait être à l'origine de l'épisode relaté par le baron de Bourgoing : à la veille de la révolution de Juillet, les rapports de Pozzo étaient plutôt rassurants, ce qui fit l'empereur dire plusieurs fois à Bourgoing : « J'ai eu de meilleures nouvelles ; j'espère qu'on ne fera pas d'imprudences. » (P. de Bourgoing, Souvenirs d'histoire contemporaine, op. cit., p. 469). 
L'empereur Nicolas semblait lire son ambassadeur avec beaucoup d'attention. Dans une lettre à son frère le grand-duc Constantin, il décrivit la situation française et en tira des conclusions tout à fait conformes à celles de Pozzo di Borgo :

«Les nouvelles de France sont bien affligeantes ; il est fort triste de penser que l'incroyable prédilection du roi pour Polignac est cause de tout cela et que le fruit de 15 années de paix non seulement est perdu, mais que nous sommes, peut-être, à la veille d'événements encore plus fâcheux et plus menaçants pour le repos du monde. Si le roi casse les chambres, il est douteux que la nouvelle élection soit meilleure; s'il renvoie son pitoyable ministère, l'on se demande comment il est possible qu'il ait lui-même provoqué un triomphe pareil pour l'opposition ! C'est pitoyable. $»^{31}$

L'empereur exposa alors à l'ambassadeur, le duc de Mortemart, sa vision de la situation française. Dans certaines publications, on trouve des allusions à un « avertissement » que Nicolas aurait envoyé au roi de France par l'intermédiaire de Mortemart. Nicolas Ier confirma lui-même l'existence d'un tel avertissement dans sa lettre au grand-duc Constantin du 6/18 août 1830, en parlant des «événements affreux survenus en France » :

«Hélas, nous l'avons prévu dès longtemps ; il n'a pas tenu à moi pour que cela n'arrive, car depuis six mois je l'ai fait écrire, j'ai fait plus, je l'ai dit haut à qui de droit, toutes mes prédictions n'ont mené à rien. Il n'y a pas un mois encore que le Roi m'a fait assurer sur sa parole que jamais il ne se permettrait des mesures illégales. $»^{32}$

Il existe d'autres témoignages (publiés) de l'avertissement écrit ou oral transmis au roi de France de la part de l'empereur russe. En 1844, Nicolas raconta à lord Aberdeen que « quelques jours avant la parution des ordonnances de juillet 1830, il avait prévenu Charles X contre toute tentative de coup d'État, dont les conséquences auraient été déplorables ${ }^{33}$. Le chancelier Pasquier mentionna à son tour dans ses Mémoires la lettre de Nesselrode à Mortemart, dans laquelle le vice-chancelier avertissait le roi Charles, de la part de l'empereur, que la Russie ne le soutiendrait pas s'il violait la Charte ${ }^{34}$. Le même Nesselrode dans une lettre à Pozzo di Borgo exprima ses craintes, et celles de l'empereur, qu'un tel « acte d'imprudence» de la part du roi pût avoir des «suites fatales »35. On trouve même des échos de cet épisode dans la presse française des années 1830 , y compris dans la presse radicale

31. Sbornik Imperatorskogo russkogo istoričeskogo ob̌̌čstva, vol. 132, Saint-Pétersbourg, 1911 ,p. 16 ; texte rédigé en français.

32. Ibid., p. 35-36.

33. S. S. Tatiščev, op. cit., p. 36, 140-141.

34. E.-D. Pasquier, Mémoires, Paris, 1895, vol. 6, pt. 2, p. 242.

35. Vnešnjaja politika Rossii..., op. cit., série n 2 , vol. 8 (16), p. 504 ; voir également Istorija vnešnej politiki Rossii (Histoire de la politique extérieure de la Russie), Moscou, 1995, p. 186 ; O. V. Orlik, Rossija v meždunarodnyh otnošenijah (La Russie dans les relations internationales), 1815-1829, Moscou, 1998, p. 60. 
et antimonarchiste, généralement hostile au « despote du Nord». Ainsi le journal Le National du 22 octobre 1839 publia la traduction de l'article du Times anglais consacré à une rencontre entre l'empereur russe et des légitimistes français. Son auteur, tout en accusant Nicolas de despotisme et de malveillance à l'égard de la France, cita néanmoins la phrase de l'empereur sur son attitude envers Charles X : «Il m'a trompé, je lui avais conseillé de ne pas violer la Charte et de respecter la loi.» Plus expressif encore est le passage du livre de Frédéric Lacroix, Les mystères de la Russie (1845). Son auteur, extrêmement critique par rapport à la Russie autocratique, accuse le tyrannique empereur de perspicacité perfide : Mortemart, selon l'auteur, ne savait rien du coup d'État de Polignac, tandis que « le tzar avait calculé la marche des choses et des événements et d'ailleurs, les rapports de ses nombreux espions ne lui laissaient aucun doute sur l'issue de la lutte engagée chez nous entre le pouvoir et le pays. Il savait un conflit inévitable et il devinait qu'il serait fatal à la branche aînée des Bourbons. »36 L'accusation d'espionnage était indispensable pour noircir l'image de Nicolas, qui apparaissait dans l'emploi inhabituel de défenseur des libertés constitutionnelles. Or les prévisions impériales ne se fondaient point sur l'espionnage (qui d'ailleurs serait bien inutile, la presse d'opposition étant remplie de prophéties sur le coup d'État), mais sur une analyse sobre et rationnelle de la situation française par l'ambassadeur russe.

Ces nombreux témoignages ne diminuent pourtant en rien la valeur du rapport de Mortemart. De première main, car rédigé immédiatement après la conversation, ce dernier permet de restituer fidèlement la pensée de l'empereur avant même les événements.

Le 16 avril 1830, Mortemart rapporte à Polignac les résultats de l'entretien confidentiel que lui accorda l'empereur. Depuis quelque temps, le monarque ne devisait en sa présence que de sujets des plus généraux. Pourtant, quatre jours auparavant, il proposa à l'ambassadeur français de faire un tour de la ville en calèche ouverte, en tête à tête, sans gardes ni valets, uniquement accompagnés par le cocher qui ne comprenait pas le français.

«En partant l'Empereur me paraissait embarrassé de commencer la conversation, il ânonnait en me questionnant, enfin il me dit, en des termes plus développés que ceux que je vais vous rendre : “Je ne sais si je dois m'adresser à l'ambassadeur de France ou au duc de Mortemart, mais mon ambassadeur à Paris m'ayant fait part d'une communication du Prince de Polignac, je dois croire que le Roi a envie de connaître mon opinion sur ce sujet. Voici le fait : Polignac a dit à Pozzo que si après avoir employé tous les moyens légaux donnés au Roi par la Constitution pour vaincre l'opposition qu'éprouvait le ministère elle ne se soumettait pas, le Roi emploierait des mesures extralégales pour la

36. F. Lacroix, Les mystères de la Russie, Paris, 1845, p. 62-63. D'ailleurs, Lacroix malgré son ton railleur reproduit fidèlement les circonstances de cette conversation que l'empereur eut avec Mortemart : «Un jour du mois d'avril 1830 il manda notre ambassadeur, M. le duc de Mortemart, l'engagea à monter en traîneau à côté de lui, et, malgré un froid piquant qui gratifia le diplomate d'un gros rhume, ils firent le tour de la capitale, tous deux seuls et n'ayant pour témoin de leur entretien que le cocher qui ne comprenait pas un mot de français. » (ibid.,p. 62). 
dompter. Ces paroles de votre ministre m'ont fait frémir et m'ont ôté l'appétit pour le restant de journée.

A-t-on bien réfléchi aux suites de pareilles mesures ? Et, dans le cas où on les emploierait, que deviendront les serments ? Que deviendront les traités ? Si, par malheur, le Roi venait à avoir besoin de l'appui de ses alliés, certes, toute ma puissance est à ses ordres, comme mon cœur dévoué à sa personne. Mais vous connaissez ces traités : si nous assurons au Roi sa couronne, nous garantissons à la France ses institutions; dans quel dédale vous nous jetteriez en sortant des voies légales ? Dites au Roi d'y bien songer ; rendez-lui mes paroles si vous les jugez sages ; il se méprendra d'autant moins au sentiment qui me les inspire que vous savez très bien que je n'aime pas les constitutions et que je ne suis nullement constitutionnel : mais le serment avant tout ; hors la foi du serment plus de gouvernement durable.

Fort de mes intentions, me dit l'Empereur, en terminant sur ce sujet, fort de mon amitié pour le Roi et de mon attachement à sa dynastie, soit que je me sois mépris ou non sur la valeur de ce que mande Pozzo, faites de mes paroles l'usage que vous croirez convenable, et rappelez-vous pour les apprécier que je ne suis pas constitutionnel." ${ }^{37}$

De nouveau, comme ce fut le cas avec La Ferronnays, Nicolas Irr $^{\mathrm{er}}$ semble avant tout se soucier de préserver le statu quo constitutionnel dans le royaume de France. Être fidèle à la loi adoptée, tel est le leitmotiv de son discours. Il admet que cette loi est relative. Personnellement, je ne suis point constitutionnaliste, souligne Nicolas, mais si les Français ont reçu la Charte (et ceci en accord avec toutes des puissances européennes suivant les traités du congrès de Vienne de 1814-1815 où fut décidé le sort de la France post-napoléonienne), leur devoir premier sera de la respecter. Cette position peut paraître étonnante à qui est habitué à voir uniquement en Nicolas un satrape-autocrate. Cependant, il existe plusieurs témoignages qui explicitent les convictions de Nicolas quant aux différentes façons de gouverner. Custine cite ce monologue prononcé par le tsar : « Je conçois la république, c'est un gouvernement net et sincère, ou qui du moins peut l'être ; je conçois la monarchie absolue, puisque je suis le chef d'un semblable ordre des choses, mais je ne conçois pas la monarchie représentative. C'est le gouvernement du mensonge, de la fraude, de la corruption [...]. » $\left(\right.$ Lettre XIII) ${ }^{38}$. Ce credo politique reçoit une formulation encore plus inattendue sous la plume de la grande-duchesse Olga, la fille de l'empereur. D'après elle, son père disait : « Je suis républicain selon mes convictions. Je ne suis monarque que par vocation. Le Seigneur me chargea de ce travail, et tant que je le

37. Archives nationales de France, 156 AP III 19, d. 1, carton 5, $\mathrm{n}^{\circ} 395$. Il est important de noter que la position de Mortemart lui-même dans l'affaire Polignac était proche de celle du tsar. Réputé «constitutionnaliste » au moment de son départ en mission à Saint-Pétersbourg, il se disait convaincu que « hors des voies légales tracées par la constitution, il n'y a plus de sécurité pour les Bourbons, ni de tranquillité pour notre pays.» (Cf. H. Contamine, Diplomatie et diplomates sous la Restauration, 1814-1830, Paris, 1970, p. 213). Son avis resta toutefois sans écho.

38. A. de Custine, La Russie en 1839, Paris, Solin, 1990, t. 1, p. 263. 
fais, je dois en être responsable. $»^{39} \mathrm{Il}$ faut mentionner également un long entretien que le tsar eut avec le baron de Bourgoing le 11/23 août 1830 (donc après la révolution de Juillet). Le lendemain le baron en fit un rapport détaillé à l'attention du comte de Jourdan (ministre des Affaires étrangères par intérim). La conversation se déroula ainsi : Bourgoing rappela à l'empereur son propre aveu prononcé lors de leur première rencontre au palais Anničkov. L'empereur disait alors que «toutes les formes de gouvernement pouvaient faire le bonheur et la tranquillité d'un peuple pourvu que la bonne foi y présidât ». Nicolas répliqua à Bourgoing (faisant allusion à son « avertissement » à l'endroit de Charles X) : « Oui, telle est bien ma profession de foi en fait de gouvernement, et si, au milieu du tumulte qui vient de régner dans Paris, on avait pillé les archives de mon ambassade, on eût été grandement surpris de voir l'autocrate de Russie charger son représentant de recommander sans cesse l'observation exacte des institutions établies et jurées. $\gg^{40}$ Nicolas répèta la même chose le 6 septembre 1830, lorsque le général Athalin, envoyé de Louis-Philippe, déplora la perfidie tyrannique de Charles X : « Oui, cela est vrai, et je le sentais si bien que j'avais toujours recommandé de marcher dans les voies constitutionnelles. Vous avez chez vous les archives, vous pouvez vous les faire ouvrir et vous y trouverez partout l'expression de cette pensée : on devait se renfermer dans la charte. $»^{41}$

Les penchants « constitutionnels » de Nicolas Irer sont mentionnés de temps en temps dans la presse française de l'époque. Ainsi un dénommé Idesbald, auteur d'un article «L'Empereur Nicolas » paru dans la Revue de Parisen 1833 rapporte la phrase attribuée au tsar : « Un gouvernement, quelle que soit sa forme, doit toujours agir avec franchise et fermeté. Un roi, s'il est constitutionnel, doit l'être sincèrement ; s'il est absolu, il doit l'être ouvertement, comme je le suis. Cette dernière façon de régner offre aussi ses difficultés ; l'absolutisme a aussi ses exigences ; la plus impérieuse est une justice constante. Si je régnais avec une constitution, rien ne m'en ferait écarter ; et c'est en la respectant le premier que je parviendrais à la faire respecter. »L'auteur de l'article parle ensuite de la lettre (sic !), que Nicolas Ier aurait envoyée à Charles X par le biais de l'ambassadeur Mortemart et qui contenait une exhortation à ne pas violer la charte ${ }^{42}$. Cet épisode fut si communément répandu, qu'il atteignit même le public russe. V. S. Pečerin répertoria dans ses Mémoires ces rumeurs, qu'il qualifia d'ailleurs de racontars, en accord avec ses

39. Nikolaj I : muž, otec, imperator (Nicolas Irr : époux, père, empereur), Moscou, 2000, p. 280 ; cf. un discours semblable de l'empereur relaté par P. D. Kiselev : « Si je devais choisir en tant que particulier une forme d'Etat pour moi-même et pour ma famille, je choisirais la république, car elle assure le mieux les garanties et la sécurité. Mais c'est un ordre qui ne peut être appliqué à tout pays ; il est approprié pour les uns et périlleux pour les autres. Dans certains cas il vaut mieux donc se tenir à ce qui a été éprouvé par le temps. » (ibid., p. 529-530)

40. AMAE, Correspondance politique. Russie, n 180, f. 294 v.; P. de Bourgoing, Souvenirs d'histoire contemporaine, op. cit., p. 517 ; N. Šil'der, Imperator Nikolaj I (L'Empereur Nicolas Ir), Moscou, 1997, vol. 2, p. 279 ; S. Tatiščev, op. cit., p. 154-155.

41. AMAE, Correspondance politique. Russie, n 181, f. 25 (relation d'Athalin du 26 août/ 7 septembre 1830).

42. Revue de Paris, 55, 1833, p. 140, 141 (voir la version manuscrite aux Archives d'État de la Fédération de Russie : GARF, f. 109, CA, op. 4, d. 298, 1. 34-45). 
convictions libérales : «Même Nicolas fut proclamé libéral. On disait que lorsque la nouvelle de la chute de Charles X atteignit le palais, le souverain fit appeler le prince héritier et lui dit : "En voilà une leçon pour toi, mon fils ! Regarde comment sont punis les rois qui ont trahi leur serment !" Et nous y croyions tout bonnement. Sancta simplicitas ! » 43

Ainsi, au cours des deux entretiens de l'empereur russe avec les ambassadeurs français, le tsar se montre défenseur de la loi constitutionnelle en France. Cet article n'a pas pour objet de traiter de la façon dont Nicolas Ier envisageait la question de la légitimité en général, néanmoins il faut souligner que ce sujet revenait sans cesse dans les conversations entre le monarque et les envoyés français, que ce fût le baron de Bourgoing, chargé d'affaires, ou bien le général Athalin, envoyé spécial de Louis-Philippe, juste après la révolution de Juillet. Devant ses interlocuteurs, l'empereur précisait invariablement qu'il comprenait parfaitement toutes les raisons incontournables qui faisaient que l'élection du duc d'Orléans au trône de France fût la meilleure issue pour le pays, mais qu'il ne pouvait pas pardonner au nouveau roi la violation du «principe de légitimité » (car selon la loi, la couronne revenait au duc de Bordeaux, petit-fils de Charles X, âgé alors de dix ans). Ce souci de légitimité n'était point exempt de motifs personnels, comme l'empereur l'indiqua lui-même au général Athalin :

«Mes sentiments pour la France sont connus, ils ne peuvent être révoqués en doute, et le caractère personnel du Roi m'inspire toute confiance. J'y trouve toutes les garanties, mais je dois le redire (ici Sa Majesté ajouta avec la plus aimable obligeance, et ne vous en fâchez pas, mon cher général), j'aurais voulu que le principe pût rester intact ; alors tout eût été bien. Je voudrais que le Roi [Louis-Philippe] fût ici et pût m'entendre : je le lui dirais avec la même sincérité. Ce principe est pour nous d'une trop haute importance, pour moi surtout, dans mes États. Il s'est présenté aussi des difficultés à mon avènement : la renonciation de mon frère n'avait pas été rendue publique, et l'acte était malheureusement resté enfoui dans des archives de famille. »44

43. Russkoje obščestvo 1830-h godov (La société russe des années 1830), Moscou, 1989 , p. 164 ; cf. l'article dans la Revue de Paris qui fait part du conseil proféré par l'empereur au gouverneur du prince héritier après l'arrivée des nouvelles de la révolution de Juillet et de la destitution de Charles X : «Profitez de cette grande leçon, Monsieur, pour enseigner à votre élève le respect que l'on doit à ses engagements, pour lui apprendre que les rois aussi sont punis quand ils manquent à leur parole. » (Revue de Paris, 55, 1833, p. 142).

44. AMAE, Correspondance politique. Russie, $\mathrm{n}^{\circ} 181$, f. 30 v.-31. Cf. la relation de Prospère de Barante, adressée au comte Molé du 30 décembre 1837, sur l'attitude de Nicolas envers LouisPhilippe : admettant que « le roi Louis-Philippe est évidemment un souverain fort habile », Nicolas n'arrive pas à lui pardonner l' " usurpation »; à son gré Louis-Philippe aurait dû rester duc d'Orléans et régent du royaume, en laissant la couronne au duc de Bordeaux. Barante explique cette intransigeance de l'empereur par les circonstances de sa propre intronisation : «L'Empereur est persuadé que l'honneur de sa vie, que ce qui doit à jamais illustrer sa mémoire, c'est d'avoir refusé la couronne jusqu'au moment où le refus du grand-duc Constantin vint rendre légitime la succession qu'avait réglée l'empereur Alexandre. C'est une circonstance de sa vie sur laquelle il aime à revenir souvent, toute inaperçue qu'elle a été en Russie, où chacun sait que le grand-duc Constantin ne voulait pas régner et où, d'ailleurs, on n'est pas accoutumé à être scrupuleux en fait d'avènement au trône. » (P. de Barante, op. cit., vol. 6, 1897, p. 64-65). 
Un détail récurrent est particulièrement digne d'attention : tous les diplomates français cités dans cet article ont remarqué la précision quasi maniaque avec laquelle Nicolas Ier définissait à quel titre et en quelle qualité se faisait l'échange entre les deux protagonistes de la conversation, lui-même et son interlocuteur. Si l'on utilise le jargon de la politologie moderne, on peut dire que le tsar se «positionnait » par rapport à son interlocuteur, et positionnait ce dernier par rapport à lui. Lors de la conversation avec Mortemart citée ci-dessus, Nicolas dit: «Je ne sais si je dois m'adresser à l'ambassadeur de France ou au duc de Mortemart», en insistant ensuite sur le fait que ses paroles sont purement informelles (ce qui est compréhensible : l'entretien tourne autour des pressentiments et des prévisions; à ce moment, rien n'a encore eu lieu qui permettrait à un monarque étranger de se prononcer officiellement). Il faut préciser que ce cas était loin d'être unique. Nicolas s'adressa avec les même précautions à La Ferronnays, qui rapporte dans une dépêche citée ci-dessus l'allocution suivante du tsar: «Ce n'est point ici l'Empereur de Russie qui se permet de faire ces observations à l'Ambassadeur de France, c'est un ami sincère du Roi qui exprime franchement des inquiétudes qui lui paraissent fondées. » Une démarche rhétorique semblable est répertoriée par le comte de Saint-Priest, « ambassadeur extraordinaire et spécial » de Charles X, dans sa dépêche du 29 janvier 1826. Lorsque le comte demande au tsar s'il peut rapporter l'opinion de celui-ci sur la question d'Orient à Charles X, Nicolas rétorque : « Très volontiers, mais n'en parlez ici à personne; c'est ici Nicolas qui parle à Charles $\mathrm{X}$ et qui vous charge de lui dire qu'il ne veut point la guerre et qu'il ne demande qu'à s'entendre avec ses alliés. $\gg^{45}$ Bourgoing, qui, une fois la nouvelle de la révolution à Paris arrivée jusqu'à Pétersbourg, fit tout son possible pour calmer l'empereur furieux et ne réussit à obtenir que la promesse de ne pas agir sans le consentement des Alliés, se heurte à la même expression de la pensée impériale : «Je vous promets de ne rien faire seul, reprit l'Empereur, et vous pouvez y compter. Quant à mon opinion personnelle, je ne puis m'en départir. Ce n'est point ici l'Empereur de Russie qui parle au chargé d'affaires du Roi de France : mais c'est moi qui en donne l'assurance à M. de Bourgoing. Je suis fort mécontent de ce qui vient de se passer, je regarde cet exemple comme dangereux et jamais je ne dissimulerai cette opinion. ${ }^{46}$

45. AMAE, Correspondance politique. Russie, $\mathrm{n}^{\circ}$ 170, f. 101-102.

46. AMAE, Correspondance politique. Russie, $\mathrm{n}^{\circ}$ 180, f. 291 v. Dans ses mémoires, Bourgoing place cette réplique de l'empereur dans un entretien précédent, celui du 27 juillet 1830 . Vers cette date-là, la révolution se met déjà en marche à Paris, mais les nouvelles n'ont pas encore atteint Saint-Pétersbourg. L'empereur ne pouvait donc être tenaillé que par un pressentiment de catastrophe imminente. Dans le texte principal, Bourgoing cite la réplique ainsi : « Oubliez que c'est l'Empereur de Russie qui s'adresse au chargé d'affaires de France ; ne voyez en moi qu'un ami de votre pays. » Dans la note en bas de page, le mémorialiste la précise une fois de plus : «Ce n'est pas l'Empereur de Russie qui interroge le représentant de la France, c'est Nicolas qui le demande à Bourgoing. » (P. de Bourgoing, Souvenirs d'histoire contemporaine, op. cit., p. 491). Le même comportement impérial transparaît dans les relations entre Nicolas et LouisPhilippe. Ayant admis à contre-cœur la monarchie de Juillet, mais considérant toujours LouisPhilippe comme un « usurpateur », l'empereur russe s'obstinait à ne pas le nommer «mon frère », appellation consacrée entre les monarques européens, ce qui soulignait une fois 
Ainsi le trait principal de Nicolas $\mathrm{I}^{\mathrm{er}}$, tel qu'il est représenté dans les deux épisodes rapportés par les ambassadeurs français, n'est pas le volontarisme despotique, conforme à sa légende posthume, mais plutôt une aspiration à respecter minutieusement les lois et les règles établies, qu'elles soient bonnes ou mauvaises, qu'elles plaisent ou non aux acteurs et aux observateurs. Les jugements moraux dépassent le cadre de cette étude. On admettra pourtant que les faits relatés ci-dessus serviront peut-être à corriger la représentation jusqu'alors figée de l'empereur Nicolas Ier.

Toujours est-il que le vaste problème de « Nicolas Ir et la France » nécessite un traitement plus ample et plus approfondi permettant de modifier et d'affiner la vision traditionnelle, voire caricaturale, de l'empereur russe, ennemi intime de la France de Juillet en particulier et de la France en général.

(traduit du russe par André Filler)

veramil@online.ru

de plus son illégitimité (cf. S. S. Tatiščev, op. cit., p. 159-160). Nicolas s'efforçait avec zèle et ostentation d'éviter à tout prix le mot «roi » en parlant de Louis-Philippe, et s'il y était contraint par l'étiquette, «c'était de mauvaise grâce et comme l'accomplissement forcé d'une formalité ». Ce n'est que le 22 octobre 1836 que Barante (en poste en Russie depuis presque un an) rapporte avec soulagement à Paris que l'empereur désigne Louis-Philippe comme le « roi de France », et cela tout naturellement et de son plein gré (cf. P. de Barante, op. cit., vol. 5, 1895, p. 487). 